\title{
INFLUENCIA DE LOS FACTORES TRANSCULTURALES EN LAS NECESIDADES Y DIAGNÓSTICOS DE ENFERMERÍA DE LA POBLACIÓN INMIGRANTE. ESTUDIO DE CASOS
}

\author{
Angela Pallarés Martí* \\ Diplomada en Enfermería y Doctora en Antropología Cultural \\ Rosa Rifà Ros* \\ Diplomada en Enfermería y Licenciada en Antropología Cultural
}

* Profesora de la Escuela Universitaria de Enfermería y Fisioterapia Blanquerna de la Universitat Ramon Llull de Barcelona

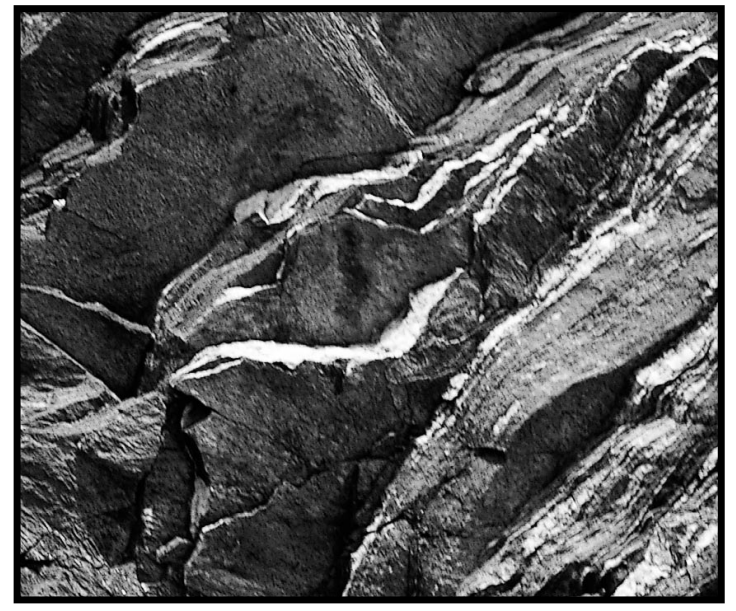

INFLUENCE OF TRANSCULTURAL FACTORS ON IMMIGRANTS POPULATIONS' NEEDS AND NURSING DIAGNOSIS

\section{ABSTRACT}

Introduction: The fact of migrations growth states the need for nursing students to acquire new knowledge regarding cultural competence.

Aims: To design nursing multicultural care models in order to offer better quality care, prevention and health education adapted to immigrant populations. To analyse, from the students nurses' perspective within the frame of Nursing and Multiculturality, an optional subject taught during their 3rd year, the immigrants' basic needs and nursing diagnosis.

Methodology: The study was carried out in two phases: exploration by students of paradigma- tic cases about migrations by means of case analysis based on the North American Nursing Diagnosis Association (NANDA) diagnosis from a transcultural perspective; and analysis of results using the program "Win Max 97 pro" to establish conceptual keys.

Results: Three stages were identified in the migration process related with needs and diagnosis: before migrating, on arrival to a country and after consolidating their situation in the country. The analysis of these cases showed that the basic needs identified by Virginia Henderson were not adequately met. Amongst all psychiatric disorders, the Ulises Syndrome stood out as the most relevant problem as a consequence of the difficulties and efforts which caused them to suffer from "chronic stress"

Conclusions: The understanding of social and cultural factors affecting immigrants, together with the analysis of Nursing Intervention Classification (N.I.C.) and Nursing Outcome Classification (N.O.C.), become an essential educational element for the professional development of future nurses facilitating the application of standarised nursing care plans.

\section{RESUMEN}

Introducción: El crecimiento de la inmigración afecta también al ámbito de las estudiantes de enfermería planteando la necesidad de adquirir nuevos conocimientos sobre competencias culturales. 
Objetivos: Diseñar modelos de cuidados enfermeros multiculturales para ofrecer una mayor calidad en las tareas de atención, prevención y educación para la salud adaptadas al colectivo emigrante. Analizar, desde la perspectiva de las estudiantes de enfermería dentro del marco de la asignatura de Enfermería y Multiculturalidad, impartida como optativa en el tercer curso de la Diplomatura, las necesidades básicas y los diagnósticos de enfermería de la población inmigrante.

Metodología: La investigación se desarrolló en dos fases: exploración de los casos paradigmáticos sobre emigración mediante el análisis de los casos por las estudiantes basados en los diagnósticos de la North American Nursing Diagnosis Association (NANDA) desde la perspectiva transcultural y análisis de los informes con el programa "Win Max 97 pro" para establecer las claves conceptuales.

Resultados: Se definieron tres etapas en el proceso de emigración relacionadas con las necesidades y diagnósticos: antes de emigrar, a la llegada a nuestro país y tras la consolidación de su situación en nuestro país. El análisis de estos casos mostró que las necesidades básicas definidas por Virginia Henderson no fueron adecuadamente conseguidas. De entre todos los trastornos psiquiátricos el Síndrome de Ulises destacó como la patología más relevante consecuencia de las dificultades y esfuerzos por los que debe de pasar en su proceso de emigración que le provoca un síndrome de "estrés crónico".

Conclusiones: La comprensión de los factores sociales y culturales de la población inmigrante combinado con el análisis de los Nursing Intervention Classification (N.I.C.) y Nursing Outcome Classification (N.O.C,), es un elemento educativo imprescindible para el desarrollo profesional de las futuras enfermeras facilitando la aplicación de los planes de enfermería de una forma estandarizada.

\section{INTRODUCCIÓN}

Uno de los cambios socioculturales más significativos de nuestro país en las últimas décadas es el haber pasado de ser emisor a receptor de inmigrantes. Esta realidad se concreta en que en los últimos 15 años la población inmigrante residente en España ha pasado de 200.000 personas a princi- pios de los años 90 a más de 1.600 .000 al inicio de la década del 2003 (Mora, 1994). A esta población censada deben añadirse un gran número de inmigrantes en situación irregular cuya cuantificación sólo puede inferirse estadísticamente. Este contexto ha cambiado la geografía humana de nuestras ciudades y evidentemente de nuestro Sistema Sanitario (Gascon, 2003).

Aunque los procesos migratorios no son un fenómeno nuevo en la historia de las sociedades en la actualidad, la creciente importancia de las desigualdades entre países a escala mundial, lejos de mitigar el problema lo han acentuado, y la imagen del inmigrante se asocia muy frecuentemente a la pobreza y la marginación.

Por esta razón es oportuno conocer, desde el ámbito de las estudiantes de enfermería de tercer curso, sus perspectivas en relación a los conocimientos sobre competencias culturales aplicadas a los cuidados enfermeros disponibles en una asignatura optativa denominada Enfermería $y$ Multiculturalidad

\section{OBJETIVOS}

Los objetivos se fundamentan en el propósito de la asignatura de "Capacitar a los estudiantes para adaptar los planes de cuidados a las diferentes características culturales de los individuos". Desde esta perspectiva se instó a las estudiantes para que:

1.- Diseñaran modelos de curas de enfermería multicultural para ofrecer una mayor calidad en las tareas de atención, prevención y educación para la salud adaptadas al colectivo emigrante.

2.- Analizaran, desde la perspectiva de las estudiantes de enfermería dentro del marco de la asignatura de Enfermería y Multiculturalidad, impartida como optativa en el tercer curso de la Diplomatura, las necesidades básicas y los diagnósticos de enfermería de la población inmigrante a partir de dos estudios de caso paradigmáticos de la emigración femenina latinoamericana (peruana) y la cada vez más visible emigración de jóvenes marroquíes.

\section{METODOLOGÍA}

La estrategia de investigación se enfocó con metodología cualitativa basada en el estudio de 
casos con el fin de que resultara útil para responder el "cómo" y "por qué" y permitiera constatar la existencia de una concepción simbólico-cultural con una gran carga de significados, estereotipos y perjuicios (Mertz, 2004).

La población incluida en el estudio fueron estudiantes de tercer curso de la Diplomatura de Enfermería $(\mathrm{n}=60)$.

Se analizaron dos estudios de casos reales:

- Ángela, cintas habladas: donde se refleja de forma autobiográfica la realidad femenina latinoamericana, resaltando el proceso migratorio desde sus inicios hasta la toma de decisión del retorno.

- Alíes Mohamed, el chico de los mil nombres: joven magrebí en pleno proceso de inserción socio-laboral.

La investigación se desarrolló en dos fases:

1. Exploración de los casos paradigmáticos sobre emigración mediante el análisis de los casos por las estudiantes basados en los diagnósticos de la North American Nursing Diagnosis Association (NANDA) (Gorajek 2003) desde la perspectiva transcultural.

2. Análisis de los informes con el programa "Win Max 97 pro" para establecer las claves conceptuales.

\section{RESULTADOS}

Desde el punto de vista de las estudiantes, la observación exploratoria descubrió tres etapas claramente diferenciadas del proceso de emigración relacionadas con las necesidades y diagnósticos (Achotegui, 2002):

- Antes de emigrar.

- A la llegada a nuestro país.

- Consolidación de su situación en nuestro país

El estudio del proceso de emigración así como del proyecto migratorio demostró en ambos casos que la preparación de la aventura migratoria, la personalidad, experiencia vital, las motivaciones para emigrar así como las necesidades de los/as emigrantes son heterogéneas dependiendo del origen del/la inmigrante, su entorno socio-cultural, económico y sus expectativas.

Se evidencia como evolucionan los-las individuos dependiendo de sus experiencias vitales. En el caso de Angela nos encontramos con una emigrante que basa el motivo de su viaje "por motivos aventureros" puesto que: "todo y tener un trabajo estable decide arriesgarse porque considera que se le presenta una oportunidad de generar ingresos que es posible que en un futuro no se le presenten y... no quiere arrepentirse".

No es el mismo caso de Mohamed, chico árabe que se va de su país, Marruecos, puesto que se da cuenta que ahí no tiene ninguna posibilidad de salir adelante pese a ser una persona con estudios universitarios.

La llegada vuelve a ser heterogénea dependiendo de la red de apoyo previa a la emigración y las posibilidades o no de conseguir una visa.

La entrada en el país vuelve a definir el estatus de emigrante, en el caso de Ángela llega con un visado de turista, dado que "unas paisanas suyas no lo consiguen y son deportadas al Perú". Fue recibida en el mismo aeropuerto por unos parientes que le dan un primer hospedaje e información/orientación durante sus primeros días de experiencia migratoria.

Mohamed atraviesa el estrecho de Gibraltar escondido en un camión, necesitando de la solidaridad cómplice del camionero para iniciar sus primeros pasos ya en el estado español, no lo espera nadie ni conoce a nadie. La desorientación máxima y la posibilidad de ser orientado por mafias en el caso de Mohamed es evidente, debiéndose de enfrentar al inicio de su experiencia migratoria a una realidad absolutamente desconocida, sin más apoyos que su intuición frente a una realidad radicalmente nueva.

Durante el proceso de consolidación vuelven a ser las experiencias vitales, el soporte familiar en destino, el cumplimiento de expectativas, las oportunidades económicas, sociales, emocionales así como la actitud del medio y las políticas de estado las determinantes del arraigo e inserción de estos nuevos conciudadanos o su expulsión e ingreso en la marginación.

Nuevamente encontramos con una situación diferenciada en el argumento vivencial de Ángela que presenta una joven con un proyecto laboral en vías de inserción laboral y social. A su llegada percibe de forma inmediata de las diferencias entre latinoamericanas y foráneas, decidiéndose por 
parecerse a las de aquí: "ahora me voy a comprar ropa como la de aquí y mañana mismito me voy a la peluquería y me quito este pelo rubio que me traje”.

Mohamed lo tiene mucho más difícil, no son sólo las diferencias físicas o el desconocimiento idiomático que imponen barreras se une el absoluto desconocimiento del medio, "al bajar del autocar a su llegada a Barcelona en la misma estación de autobuses pidió consejo a "colegas" de emigración que exclusivamente lo alertaron frente a la policía. Sólo después de deambular por las calles durante diversos días contacto con una asociación de ayuda al emigrante, iniciando a partir de ese momento su inserción laboral y social.

El colectivo inmigrante está formado personas sanas para poder enfrentarse con posibilidades de éxito al proceso migratorio. Sólo así se comprende la relativa poca incidencia de patologías graves entre este grupo.

Los dos casos estudiados demostraron que los emigrantes no consiguen tener sus necesidades básicas cubiertas, ya sea por incapacidad de acceder a ellas (falta de alojamiento en el caso de Mohamed) o por el choque cultural y/o barrera idiomática que imposibilita una adecuada comprensión del nuevo entorno.

El análisis de estos casos mostró a juicio de las estudiantes que de las 14 necesidades básicas definidas por Virginia Henderson no fueron adecuadamente conseguidas por diferentes razones en cada caso (Evers, 2000):

1. Necesidad de dormir y descansar: para Ángela estas necesidades se ven parcialmente cubiertas, aunque con dificultades, el exceso de trabajo así como el estrés provocado al caducársele los papeles le provoca una alteración.

\section{Necesidad de comer y beber adecuada-} mente: para los dos casos existen condicionantes que alteran la posibilidad de cubrir esta necesidad, pero con grandes diferencias. En el caso de Ángela debe de adaptarse a los nuevos alimentos que encuentra al llegar a su nuevo destino pero no padece en ningún momento penuria alimentaria. Como ocurre en el resto de necesidades, Mohamed padece hambre y grandes penurias durante la primera etapa de inserción en su experiencia migratoria en el estado español.

\section{Necesidad de mantener la higiene corpo-} ral e integridad de la piel: Mohamed, al principio de su estancia en Barcelona, no tiene posibilidad de una correcta higiene al no disponer de un domicilio debiendo de pernoctar en la calle. Ángela al ser recibida por sus parientes tiene esta necesidad cubierta desde un inicio.

\section{Necesidad de actuar según creencias y} valores: es importante valorarlo. En principio Ángela se encuentra en un entorno de acogida que le permite continuar con sus valores; no ocurre igual con Mohamed que a las dificultades ya tratadas se encuentra con un entorno que dificulta proteger sus valores y creencias.

5. Necesidad de comunicarse: Ángela ensaya un sistema muy creativo para contactarse con sus padres, graba cintas donde les relata algunos aspectos de su vida en Barcelona; siente que necesita oír la voz de sus padres para sentirse amparada. Además tiene la necesidad de nuevos amigos, difícil de conseguir debido a su situación laboral.

6. Necesidad de evitar peligros: la falta de "papeles" de Mohamed le impedían transitar por las calles con libertad. Ángela al caducar su visado se encontró de la noche a la mañana en una situación difícil llena de angustia y soledad. Angela se sentía fugitiva de la ley cuando veía un patrullero pensaba que venia a por ella. Estos pensamientos le afectaban mucho psicológicamente y socialmente".

Estas carencias pueden llegar a provocar en los/las emigrantes situaciones diagnósticas que abarcan áreas, físicas, sociales y psicológicas observando a sus pacientes de forma integral, del estudio de casos se observó como los diagnósticos de enfermería básicamente se centraban en:

1. Ansiedad relacionada por el cambio en su estilo de vida.

2. Una relativa mala adaptación relacionada con la incapacidad de integración en su nuevo entorno.

3. Sentimiento de no sentirse adaptados/as.

4. Riesgo de deterioro de la comunicación verbal, por desconocimiento del idioma.

5. Riesgo de infecciones, relacionado con los hábitos y el difícil acceso a la información de la asistencia sanitaria. 
6. Deterioro en ocasiones de su capacidad de aprendizaje.

Mayoritariamente los diagnósticos de enfermería entre el colectivo emigrante se basaron en los problemas subyacentes a diferencias culturales fundamentales como el control de la incertidumbre, la diferente percepción y la autopercepción del binomio individuo/colectivo, la distinción de género y la diferencia de trato a la hora de entender la distancia jerárquica.

Como consecuencia a estos diagnósticos la asistencia sanitaria debería abarcar las dimensiones físicas, sociales y psíquicas a fin de prevenir o tratar las alteraciones que presenta este colectivo.

En cuanto a la dimensión física básicamente el esquema que se siguió fue:

- Prevención de patologías comunes.

- Programa de atención específica: mujeres y adolescentes.

- PAPPS (programa de actividades preventivas y de promoción de la salud): actividades preventivas.

- Seguimiento de las patologías que hubiera podido importar.

Respecto a la dimensión social se tuvo en cuenta un principio básico desde la perspectiva de la enfermería transcultural que "los grupos humanos responden a la enfermedad de forma diferente según sea su estructura, su sistema de valores, las

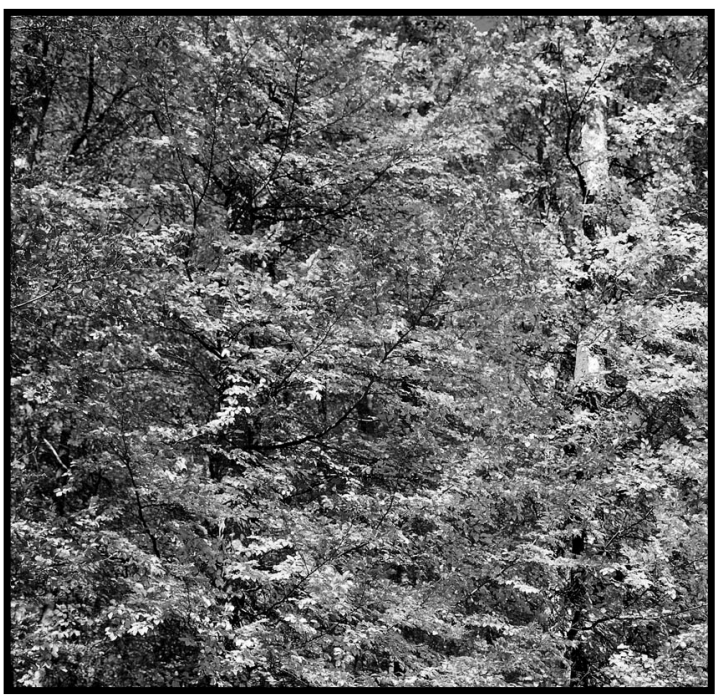

relaciones sociales y su cultura”, por lo tanto la enfermería debe de adaptarse a la cosmovisión de cada uno de sus posibles interlocutores (Bellabes, 2004).

La dimensión psíquica es frecuentemente la mas afectada sin embargo en ocasiones es difícil de desenmascarar, debido a que los enfermos en la consulta verbalizan trastornos diversos ocultando el verdadero origen de su patología. Esquemáticamente las patologías psiquiátricas más frecuentemente diagnosticas por enfermería fueron la depresión debido al cambio en los estilos de vida y soporte familiar (ejemplo), la ansiedad producto de las dificultades económicas y de vivienda o los trastornos psicosomáticos, producto de los obstáculos para legalizar su situación.

De entre todos los trastornos psiquiátricos el Síndrome de Ulises (Sindrome de Ulises e inmigrantes) destacó como la patología más relevante consecuencia de las dificultades y esfuerzos por los que debe de pasar en su proceso de emigración que le provoca un síndrome de "estrés crónico".

\section{DISCUSIÓN}

En el meta-análisis de los estudios de casos, las estudiantes de enfermería resaltan la existencia de factores clave como la buena salud física de la mayoría de los emigrantes y el duelo migratorio desde la perspectiva de los cuidados de enfermería al que les es muy difícil sobreponerse.

El $75 \%$ de las estudiantes reconoció que aumento su sensibilidad hacia la emigración a partir de la profundización de las claves sociales y culturales en los estudios de casos, la respuesta de las alumnas a esta problemática se puede resumir en que desde el punto de vista de la enfermería, el multiculturalismo constituye una gran oportunidad para constatar la universalidad de los cuidados y su diversidad de manifestaciones culturales.

Así pues la comprensión de los factores sociales y culturales de la población inmigrante combinado con el análisis de los Nursing Intervention Classification (N.I.C.) y Nursing Outcome Classification (N.O.C,) (Torre 2003) es un elemento educativo imprescindible para el desarrollo profesional de las futuras enfermeras facilitando la aplicación de los planes de enfermería de una forma estandarizada. 
Finalmente es importante resaltar que para la mayoría de las alumnas representa el punto de inicio de un cambio personal en la percepción, no sólo de salud y por lo tanto como profesional, sino como personas de aquéllos que antes para ellas eran sólo inmigrantes, asumiendo que el enriquecimiento en la comprensión de las nuevas realidades se produce tras la adquisición, por parte de las alumnas, de estos nuevos conocimientos que aportan elementos para conseguir la competencia cultural de los profesionales (Betancourt, 2004).

\section{BIBLIOGRAFÍA}

- Achotegui, J. (2002) La depresión en los inmigrantes: una perspectiva transcultural. Mayo. Barcelona.

- Bellabes, M., Borschnek, M., Bouazouz, H., Chiri, C., Csinidis, C., Daubie, P., Garino, M., Favre, J., Nouri, W., and Sambati, L. (2004) Cultural approach to care. Soins (686 PT1): 30-33, 2004.

- Betancourt, J.R. (2004) Cultural competence-marginal or mainstream movement? N. Engl. J. Med. 351 (10): 953-995.
- Evers, G.C. (2000) Clinical nursing research. New standars for quality assurence for nursing and patient. Pflege . 13 (3): 133-138.

- Gascon , C. (2003) Infectous diseases and inmigration. Enfer. Infecc. Microbiol. Clin 21 (10): 535-539.

- Goraje-Jozwik, J. (2003). The Classification systems of nursing practice-the historical and practical perspective. Ann Uni Mariae. Curie Sklodowska [Med.]58 (2): 202-207.

- Mertz, C., Belbab, H., Chabriere, N., Faucon-Artin, H., Lecam, S., Legrand, V., Margarie de, C.

- Mora, E. Norman. (1994) Population projection and its principal components: the future model of population in the province of Alicante. Bol.Asoc. Demogra. Hist 12 (1): 55-80.

- Sindrome de Ulises e inmigrantes.

http://www.entornosocial.es/sociosanitaria/so56140 3.html

- Torre, R., Bertazzoni, G. (2003) Nursing Intervention Classification (N.I.C.) and Nursing Outcome Classification (N.O.C.) of lowa University: a description of methods, tools and contents. Prof. Inferm. 56 (3): 143-158.

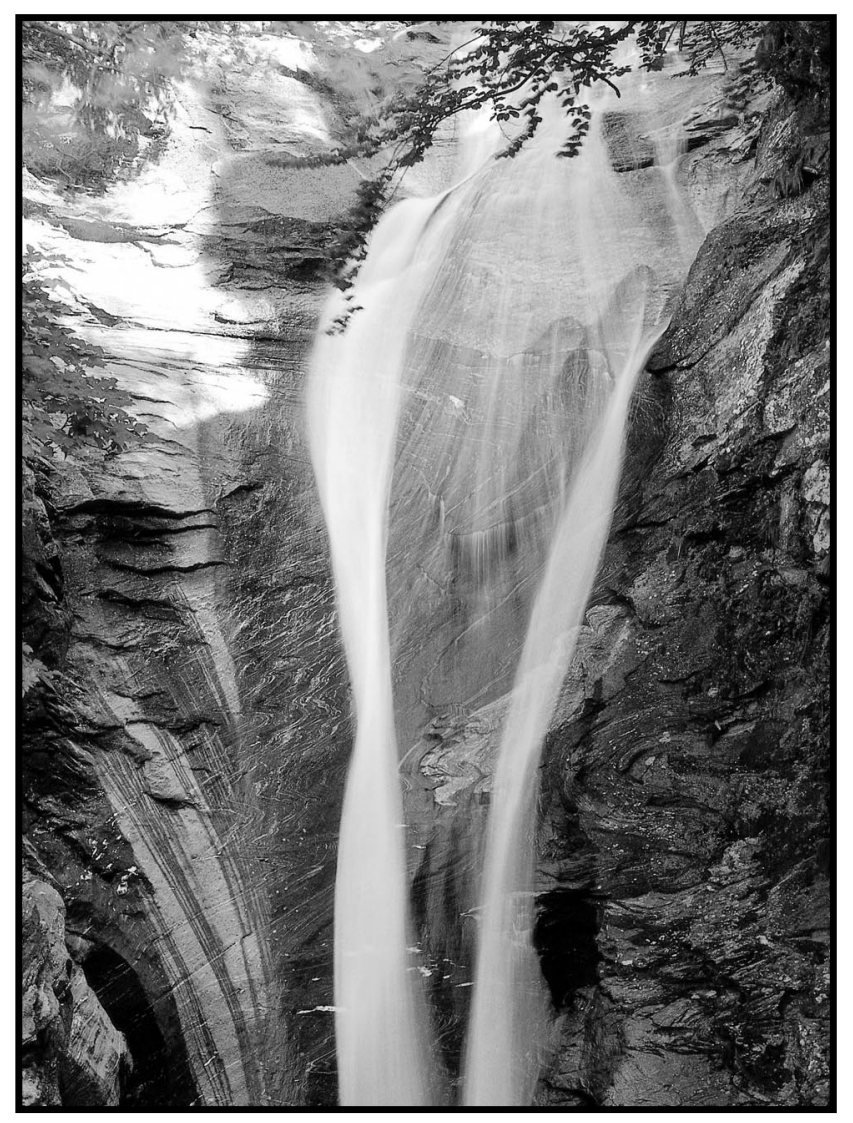

